\title{
INVITED COMMENTARY
}

\section{New somatostatin analogs: will they fulfil old promises?}

\author{
S W J Lamberts, A J van der Lely and L J Hofland \\ Department of Medicine, Erasmus Medical Centre, 40 Dr. Molewaterplein, 3015 GD Rotterdam, The Netherlands
}

(Correspondence should be addressed to S W J Lamberts; Email: lamberts@inw3.azr.nl)

\section{Introduction}

A peptide inhibiting the release of growth hormone (GH) was originally detected accidentally during studies of the distribution of GH-releasing factor in the hypothalamus of rats (1). This peptide, called somatostatin, proved to be a cyclic peptide consisting of 14 amino acids. Subsequent studies have considerably expanded this initially simple concept of somatostatin as a peptide whose main function is the regulation of $\mathrm{GH}$ secretion. Somatostatin is present and plays an inhibitory role in the regulation of several organ systems in man and other species, such as the central nervous system, the hypothalamus and the pituitary gland, the gastrointestinal tract, the endocrine and exocrine pancreas, several components of the immune system, the retina, and vessel walls $(2,3)$ Apart from the inhibition of GH and thyrotropin (TSH) secretion, somatostatin also inhibits a variety of other physiological functions such as gastrointestinal motility, gastric acid production, pancreatic enzyme secretion, bile and colonic fluid secretion. It inhibits the secretion of hormones such as insulin, glucagon, secretin, and vasoactive intestinal polypeptide $(2,3)$. In these different activities somatostatin acts as a neurohormone, a neurotransmitter, or as a local factor acting via autocrine or paracrine mechanisms. In his Nobel lecture, Guillemin summarized a number of these newly discovered mechanisms (4). In view of the ability of somatostatin to inhibit such a variety of physiological processes, he predicted that this peptide might be of therapeutic value in clinical conditions involving hyperfunction of the organ systems mentioned above. However, the multiple simultaneous effects of pharmacological concentrations of somatostatin in different organs, the need for intravenous administration, its short half-life in the circulation, and the post-infusion hypersecretion of hormones considerably hampered the initial enthusiasm, as well as its clinical use.

\section{The development of somatostatin analogs}

In 1974, two years after the discovery of somatostatin, the Sandoz Company in Basel, Switzerland started a program to develop long-acting analogs. Using stepby-step modification of the conformationally stabilized, central, essential part of the somatostatin molecule, Bauer et al. succeeded in synthesizing an analog which did not have most of the disadvantages of the native peptide (5). In monkeys this analog, code named SMS 201-995 (octreotide; H-[D]-Phe-Cys-Phe[D]-Trp-Lys- Thr-Cys-Thr(OL)), inhibits the secretion of $\mathrm{GH}$, glucagon and insulin 45, 11 and 1.3 times more actively, respectively, than native somatostatin. The analog was highly resistant to enzymatic degradation (half-life about $2 \mathrm{~h}$ after subcutaneous administration), and the subcutaneous administration of $50 \mu \mathrm{g}$ of the drug in acromegalic patients suppressed $\mathrm{GH}$ for $6-8 \mathrm{~h}$, but was not followed by rebound hypersecretion of GH (6).

In the subsequent years, large numbers of somatostatin receptors were discovered on most human neuroendocrine tumors (7). These high-affinity receptors were particularly densely and homogeneously distributed over the GH-secreting pituitary tumors of acromegalic patients and in carcinoid and pancreatic islet cell tumors. Long-term therapy with octreotide was demonstrated to control hormonal hypersecretion successfully in about $65 \%$ of acromegalic patients (8). Apart from instant clinical improvement, notable tumor shrinkage was also demonstrated in most patients (9). Also, in patients with metastatic carcinoid disease and islet cell tumors, octreotide therapy in most instances ameliorated most of the clinical problems (10). Control of symptoms, such as diarrhea and flushing attacks, caused by an overproduction of serotonin or tachykinin(s), was reported in 70 to $90 \%$ of patients with metastatic carcinoid tumors. Diarrhea, dehydration, and hypokalemia in patients with tumors secreting vasoactive intestinal polypeptide, and peptic ulceration, hypoglycemic attacks, and necrolytic skin lesions in patients with tumors secreting gastrin, insulin and glucagon, respectively, were well-controlled in $50-80 \%$ of patients treated with octreotide. Apart from the instant improvement in quality of life, a prolonged survival in octreotide-treated patients with metastasized carcinoids and islet cell tumors was ascribed, at least in part, to a temporary inhibition of tumor growth (10).

Octreotide had initially to be administered subcutaneously two or three times daily. A long-acting form was eventually developed which consists of 20 to $30 \mathrm{mg}$ octreotide mixed with microspheres of 
DL-lactide-co-glycolide polymer, which delivers therapeutic octreotide levels for at least 28 days after intramuscular injection. Also two other cyclin analogs of somatostatin with only slightly different activity profiles, vapreotide (RC-160), and lanreotide (BIM23014) are currently used. A slow-release formulation of lanreotide is available, which is active for 7-14 days, while a subcutaneous autogel-preparation of 90-120 mg lanreotide with an even longer duration of activity has recently been introduced for clinical purposes in several countries.

The high density of somatostatin receptors on some tumors, as well as the availability of potent and stable somatostatin analogs as radioligands, made it possible to use somatostatin-receptor scintigraphy with ${ }^{123} \mathrm{I}-$ $\mathrm{Tyr}^{3}$-octreotide, and subsequently with ${ }^{\mathrm{III}} \mathrm{ln}$-labeled pentetic acid-D-Phe ${ }^{1}$-octreotide for the visualization of somatostatin receptor-positive tumors in man (11, 12). The demonstration of an effective internalization of the somatostatin receptor complex by human tumors was the basis for the subsequently developed concept of targeted therapy with somatostatin analogs coupled to cytotoxic agents or radionuclides $(13-15)$.

\section{Indications and adverse effects}

Today, more than 15 years after the clinical introduction of octreotide, one cannot deny that there is a certain disappointment concerning the limited number of proven indications for the use of somatostatin analogs in clinical medicine. Apart from acromegaly, metastatic carcinoid disease, and Vipomas, only acute variceal bleeding of the esophagus and the perioperative protection around pancreatic surgery have been approved as indications for the use of octreotide in a number of countries. Unfulfilled promises for its use in the glycemic and metabolic control of diabetes, in upper gastro-intestinal bleeding, secretory diarrhea, pancreatitis, and especially in the treatment of cancers, like those of the breast, prostate and colon have in fact dampened the enthusiasm for research with somatostatin analogs in this field.

Still, integration of a number of previous and recent observations might refuel the enthusiasm for renewed studies of the potential value of somatostatin analogs in the major and commonly occurring diseases mentioned above.

Early studies demonstrated that long-term administration of somatostatin analogs, like octreotide, in most instances only transiently inhibit physiological actions, either because of their desensitizing actions, or because of a compensatory reaction of local stimulatory factors which, in most instances, eventually have an overriding effect (16). After seven days of octreotide administration (100-300 $\mu \mathrm{g} /$ day), the initial inhibitory effects on secretion of gastric acid, amylase, trypsin and lipase diminish or even disappear
$(17,18)$. This desensitizing or adaptational phenomenon explains why long-term treatment with octreotide causes so little adverse effects: in most cases the initially occurring symptoms such as cramps, diarrhea, and fatty stools disappear within 10-14 days of continuous octreotide administration, probably as a consequence of local adaptation in the gastrointestinal tract and exocrine pancreas. Only the effects on the composition of the bile and the contractility of the gall bladder are of longer duration and cause gall stone formation during long-term somatostatin analog administration in $10-30 \%$ of individuals. Fortunately, clinically symptomatic gall bladder disease occurs in only about $1 \%$ of individuals per treatment year on octreotide. So, it seems that the statement 'each disadvantage has an advantage' (Johan Cruyff) also applies to octreotide: the rapid adaptation to octreotide is favorable with regard to its adverse effects and safety, but it limits its successful use in the treatment of patients with functional disorders of the gastro-intestinal tract and the pancreas: short-term therapy with octreotide transiently lowers the blood pressure in the abdominal vessels, improving the control of acute variceal bleedings, while it transiently suppresses pancreatic enzyme production, improving the outcome of elective pancreatic surgery. However, it turned out to be much more difficult to prove effectiveness of octreotide treatment on secretory diarrhea, or in the closure of intestinal fistulas. Despite the obvious initial clinical improvement in most cases, these initial effects seem to level off during longer octreotide therapy.

\section{Somatostatin receptor subtypes}

With regard to a potential role of somatostatin analogs in the glycemic and metabolic control of diabetes mellitus and in the treatment of cancer, basic research in the field of somatostatin seems to offer at least some explanation for the marginal efficacy of octreotide treatment. The various actions of somatostatin are mediated through specific membrane receptors, which have been demonstrated in all target organs of somatostatin, such as the brain, the anterior pituitary, endocrine cells of the gastro-intestinal tract and the pancreas, some immune cells and the retina. At least five subtypes of the human somatostatin receptor have been cloned and characterized (3). These subtypes (sst) are identical in 42 to $60 \%$ of their amino acid sequence, and belong to a superfamily of receptors with seven membranespanning domains. The genes for these somatostatin receptor subtypes are located on different chromosomes, suggesting different functions in different organs. Indeed, distinct, but overlapping patterns in the expression of these different somatostatin receptor subtypes have been demonstrated. All five subtypes are functionally linked to adenylate cyclase through a coupling mechanism involving guanine nucleotide- 
binding (G) proteins. But apart from inhibition of adenylyl cyclase activity, the different subtypes are linked to multiple other cellular effect or systems, including effects on phosphotyrosine phosphatases, STAT 5 and MAP kinases, inhibition of $\mathrm{Ca}^{2+}$-influx, activation of phospholipase $\mathrm{A}$ and $\mathrm{C}$, as well as others (3).

Although very preliminary, some specific physiological regulatory roles might be attributed to these somatostatin receptor subtypes: $\mathbf{s s t}_{2}$ and $\mathrm{sst}_{5}$ are involved in the control of $\mathrm{GH}$ release; sst $_{5}$ seems of importance in the control of insulin, and possibly glucagon secretion; sst $_{3}$ and to a lesser extent sst $_{2}$ can induce apoptosis; sst $_{1}$ and sst $_{5}$ have an inhibitory effect on the cell cycle; sst $_{1}$ might be involved in angiogenesis; the function of $\mathrm{sst}_{4}$ is largely unknown.

All five somatostatin receptor subtypes bind natural somatostatin-14 with a similar high affinity, but there are major differences in the binding affinities of the different structural analogs of somatostatin. Octreotide, lanreotide, and vapreotide bind with a high affinity to $\mathrm{sst}_{2}$, and to a lesser extent to $\mathrm{sst}_{5}$, have a low affinity for $\mathrm{sst}_{3}$, and do not bind to $\mathrm{sst}_{1}$ and $\mathrm{sst}_{4}$. In retrospect, this is not surprising: octreotide was selected from a number of other analogs because of its preferential GH-inhibitory effect, while it hardly affected insulin release, which is considered to be mainly regulated by sst $_{5}$ (10).

\section{New developments in somatostatin receptor physiology}

In recent years major new developments have been reported which have helped in the understanding of somatostatin receptor physiology. The availability of sst $_{5}$-specific, high affinity sst $_{2}$ and mixed sst $_{2^{-}}$and sst $_{5}$-specific analogs has allowed Melmed's group to delineate the role of $\mathrm{sst}_{2}$ and $\mathrm{sst}_{5}$ in the regulation of pituitary hormone secretion $(19,20)$. Using primary human fetal pituitary cell cultures it was demonstrated that GH secretion was suppressed equally by analogs preferential for either $\mathrm{sst}_{2}\left(\mathrm{IC}_{50}\right.$ for receptor binding affinity, $0.19-0.42 \mathrm{nmol} / \mathrm{l})$ or $\mathrm{sst}_{5}\left(\mathrm{IC}_{50}, 0.37 \mathrm{nmol} / \mathrm{l}\right)$. Similar analogs with high affinity for $\mathrm{sst}_{2}$ or $\mathrm{sst}_{5}$ decreased TSH secretion by 30-40\% $(P<0.05)$. These results demonstrated, for the first time, that not only $\mathrm{sst}_{2}$, but also $\mathrm{sst}_{5}$ is critical for the physiological regulation of GH and TSH (19). Subsequently, using primary cell cultures prepared from human GH secreting pituitary tumors, these investigators demonstrated that heterologous analog combinations containing both sst $_{2^{-}}$and sst $_{5}$-selective compounds were more potent in decreasing $\mathrm{GH}$ release than these analogs used alone, or than combinations of compounds specific for the same receptor subtype. In pure prolactin (PRL) secreting human pituitary tumor cells hormone release was suppressed by $\mathrm{sst}_{5}$-selective, but not by sst $_{2}$-selective analogs (20).

New fundamental insights into somatostatin receptor physiology were presented in 2000 by Patel's group $(21,22)$. Using $\mathrm{CHO}_{-} \mathrm{K}_{1}$ transfected cells, these investigators demonstrated ligand-induced somatostatin receptor dimerization. Both natural somatostatin and somatostatin analogs could produce homo- or heterodimerization of the somatostatin receptor subtypes 1 and 5. Such a ligand-induced dimerization process resulted in increased binding affinity and modified somatostatin receptor subtypes (22). This means that protein interaction between different members of the somatostatin receptor subfamily are involved in a molecular cross-talk. However, a cross-talk between different related $\mathrm{G}$ protein-coupled receptor families resulting in enhanced functional activity was also subsequently demonstrated. Again, using $\mathrm{CHO}_{1} \mathrm{~K}_{1}$ cells it was demonstrated that dopamine receptor $\mathrm{D} 2 \mathrm{R}$ and sst $_{5}$ interact physically through hetero-oligomerization to create a novel receptor, which also has an enhanced functional activity (21).

After these breathtaking new insights into somatostatin receptor physiology, the practical consequences of these observations rapidly became clear. Melmed's group which demonstrated earlier that somatostatininduced inhibition of $\mathrm{GH}$ secretion from normal human pituitary cells is mediated through both $\mathrm{sst}_{2}$ and $\mathrm{sst}_{5}$, subsequently demonstrated that combined addition of $\mathrm{sst}_{2}$ - and $\mathrm{sst}_{5}$-selective agonists resulted in a synergic suppression of $\mathrm{GH}$ releasing hormone (GHRH)-stimulated $\mathrm{GH}$ release from primary fetal pituitary cells, achieving 73\% inhibition, which is much higher than attained with the agonists alone $(32 \%$ and 34\% respectively; $P<0.05$ ) (23). These observations suggest that simultaneous activation of $\mathrm{sst}_{2}$ and $\mathrm{sst}_{5}$ induced a functional association of these receptor subtypes, resulting in a synergistic suppression of GH secretion. In subsequent studies by Jaquet's group in human $\mathrm{GH}$-secreting pituitary tumors the functional consequences for optimizing the medical treatment of acromegaly became apparent (24). These investigators quantified $\mathrm{sst}_{2}$ and $\mathrm{sst}_{5} \mathrm{mRNA}$ expression and the in vivo sensitivity of $\mathrm{GH}$ release to octreotide in 10 pituitary tumors from acromegalic patients. The octreotide-sensitive $\mathrm{GH}$ secretory adenomas presented with a high level of both $\mathrm{sst}_{2}$ and $\mathrm{sst}_{5}$ mRNA expression. However, among 5 tumors which were only partially sensitive to octreotide, sst $_{2}$ mRNA expression was ninefold lower, and sst $_{5}$ mRNA expression approximately sevenfold higher than in the octreotide-sensitive tumors. In this last group of partially octreotide-sensitive tumors both the sst $_{5}$-preferential compound BIM-23268, but especially the $\mathrm{sst}_{2}$ and sst $_{5}$ bi-specific compound, BIM-23244, were quite effective in suppressing GH secretion. Similarly BIM23244 was able to suppress PRL release from five mixed GH/PRL-secreting adenomas by more than 
$50 \%$. These data indicate that due to the heterogeneous expression of $\mathrm{sst}_{2}$ and $\mathrm{sst}_{5}$ receptor subtypes in $\mathrm{GH}$ secreting adenomas, a bispecific analog, such as BIM23244 that can activate both receptors, achieves better control of $\mathrm{GH}$ hypersecretion in a larger number of acromegalic tumors than octreotide (24).

The new fundamental insights in somatostatin receptor physiology opening the concept of a multi-familial cross-talk which induces new possibilities to regulate cellular hormonal sensitivity was recently further demonstrated by the synthesis of a somatostatin-dopamine hybrid molecule, BIM-23A387 by BioMeasure, which has a high affinity in the nmol/l range for both $\mathrm{sst}_{2}$ and the dopamine $\left(\mathrm{DA}_{2}\right)$ receptor. Indeed, such a hybrid molecule was demonstrated in vitro to have an enhanced effect on PRL release. This is only the start of the search for new therapeutic opportunities, as the combination in one molecule of somatostatin analogs with opiates and/or cytokines is now also theoretically possible (J P Moreau, personal communication).

The simultaneous expression of multiple somatostatin receptor subtypes on the same cells in target organs, as well as many somatostatin receptor-positive tumors indicates that a differential coupling via the 5 sst subtypes which activate simultaneously a variety of intracellular signaling systems are an essential part of somatostatin function. The interactive, multireceptor responses which now also include homo- and heterodimerization and cross-talk at the membrane level with other $\mathrm{G}$ protein-coupled systems is a tantalizing new concept for drug designers.

\section{A universal ligand: SOM-230}

In this evolving picture, the Novartis company has now introduced SOM-230, a universal compound (25). By using a stunning technology (alanine scanning) certain functional groups which confer affinity for the five somatostatin receptor subtypes were transposed into a cyclohexapeptide template. Using membranes from sst-transfected cells for receptor binding studies, the in vitro model of $\mathrm{GH}$ release by rat pituitary cells, as well as in vivo studies of $\mathrm{GH}$, glucagon and insulin release in rats, dogs and monkeys, Bruns and his group of investigators succeeded in synthesizing SOM-230, a compound which binds with a high affinity to $\mathrm{sst}_{1}$, $\mathrm{sst}_{2}, \mathrm{sst}_{3}$ and $\mathrm{sst}_{5}$, and with a lower affinity to $\mathrm{sst}_{4}$ (25). In this sense, one can indeed speak of a virtual 'universal' ligand. In rats, dogs, and rhesus monkeys, SOM-230 potently and dose-dependently decreases insulin-like growth factor-I (IGF-I) levels for prolonged periods of time up to 120 days. No desensitization of the suppressing effect of SOM-230 on IGF-I levels was observed, which is in contrast to what has been seen with octreotide in man. An explanation for this discrepant effect between SOM-230 and octreotide seems to be the different intracellular dynamics of $\mathrm{sst}_{2}$ and $\mathrm{sst}_{5} . \mathrm{sst}_{2}$ is rapidly internalized after ligand binding, but $\mathrm{sst}_{5}$ demonstrates early recycling after internalization, with massive re-recruitment from intracellular stores (back) to the membrane (26). Additional characteristics of SOM-230 include a very favorable T $1 / 2$ of nearly $24 \mathrm{~h}$, as well as the preliminary evidence that glucose levels in rats and dogs remain normal during long-term administration, despite the powerful sst $_{5^{-}}$ mediated suppression of insulin release.

What will happen now in the field of somatostatin? A logical direct approach for both the producers of BIM-23244 (sst $2^{-}$and sst $_{5}$-specific) and SOM-230 (universal ligand) is to start phase I trials to investigate the pharmacokinetics, toxicity and dose-range of these new compounds. The next step will probably be a phase II trial in acromegalic patients (both octreotidesensitive and octreotide-insensitive) as a proof of principle that these compounds are effective and well tolerated during long-term therapy. Although twothirds of acromegalic patients can be biochemically 'cured' with the currently available somatostatin analogs octreotide and lanreotide, the recent data of a high expression of $\mathrm{sst}_{5} \mathrm{mRNA}$, which seems to give rise to high $\mathrm{sst}_{5}$ receptor expression, especially in octreotide-resistant acromegalic patients, suggests that the addition of SOM-230 and/or BIM-223244 to the therapeutic arsenal for the medical treatment of acromegaly will probably complement their efficacy to close to $100 \%$ of acromegalics, including those tumors that are mixed mammo-somatotropic. Also dopamine-resistant or -intolerant patients with prolactinomas will probably benefit from treatment with these compounds. It will be interesting to see what the eventual position of the GH-receptor antagonist Pegvisomant will be in this field. This compound has the disadvantage that it does not exert a direct inhibitory action on the pituitary tumor.

SOM-230 can also, at last, give an answer to the long-standing question, whether the $\mathrm{sst}_{1^{-}}$and $\mathrm{sst}_{3^{-}}$ mediated anti-tumor effects (cell cycle inhibition, induction of apoptosis) have a clinically beneficial effect not only in patients with inoperable carcinoids and islet cell tumors, but also in patients with otherwise non treatable somatostatin receptor-positive breast, prostate and colonic cancers, as well as malignant lymphomas. Careful analysis of the somatostatin receptor subtype distribution on the tumors of the patients included in such clinical trials is mandatory, however. But apart from these potentially important direct antiproliferative actions of SOM-230, the manipulation of IGF-I bioactivity might also have new, important clinical significance in the control of tumor growth in a variety of solid cancers.

Finally, the use of somatostatin analogs in diabetes mellitus remains an open, but exciting field. The longterm control of $\mathrm{GH}$ and IGF-I secretion might be of benefit in improving overnight metabolic control in type I diabetics by suppressing GH release, and in the 
prevention of the at least partially IGF-I-dependent diabetic complications in the eye and kidney as well (2730). In previous studies slightly initial beneficial, but often transient effects were observed during octreotide administration which, at least in part, were attributed to the transient and marginal suppression in circulating IGF-I concentrations (29). Careful, placebocontrolled randomized clinical trials will give answers concerning the potential value of SOM-230 in the treatment of functional gastro-intestinal disorders such as fistulas, and in secretory diarrhea as well.

Next year it will be 30 years after the characterization of somatostatin. A dazzling story involving contributions from basic and clinical science, as well as heavy involvement of industry bring the story of this peptide in a full circle back to the prediction of Guillemin in his Nobel lecture (4).

\section{References}

1 Brazeau P, Vale W, Burgus R, Ling N, Butcher M, Rivier J et al. Hypothalamic polypeptide that inhibits the secretion of immunoreactive pituitary growth hormone. Science 1973179 77-79.

2 Lamberts SWJ, Krenning EP, Klijn JGM \& Reubi JC. Clinical applications of somatostatin analogs. Trends in Endocrinology and Metabolism 19901 139-144.

3 Patel YC. Somatostatin and its receptor family. Frontiers in Neuroendocrinology 199920 157-198.

4 Guillemin R. Peptides in the brain: the new endocrinology of the neuron. Science 1978202 390-402.

5 Bauer W, Briner U, Doepfner W, Haller R, Huguenin R, Marbach P et al. SMS 201-995: a very potent and selective octapeptide analogue of somatostatin with prolonged action. Life Sciences 1982 31 1133-1140.

6 Lamberts SW, Oosterom R, Neufeld M \& del Pozo E. The somatostatin analog SMS 201-995 induces long-acting inhibition of growth hormone secretion without rebound hypersecretion in acromegalic patients. Journal of Clinical Endocrinology and Metabolism 198560 1161-1165.

7 Reubi JC, Maurer R, von Werder K, Torhorst J, Klijn JG \& Lamberts SW. Somatostatin receptors in human endocrine tumors. Cancer Research 198747 551-558.

8 Lamberts SW, Uitterlinden P, Verschoor L, van Dongen KJ \& del Pozo E. Long-term treatment of acromegaly with the somatostatin analogue SMS 201-995. New England Journal of Medicine 1985 $3131576-1580$.

9 Lamberts SW. The role of somatostatin in the regulation of anterior pituitary hormone secretion and the use of its analogs in the treatment of human pituitary tumors. Endocrine Reviews $19889417-436$.

10 Lamberts SW, van der Lely AJ, de Herder WW \& Hofland LJ. Octreotide. New England Journal of Medicine 1996334 246-254.

11 Krenning EP, Bakker WH, Breeman WA, Koper JW, Kooij PP, Ausema L et al. Localisation of endocrine-related tumours with radioiodinated analogue of somatostatin. Lancet $1989 \mathbb{1}$ $242-244$.

12 Lamberts SW, Bakker WH, Reubi JC \& Krenning EP. Somatostatinreceptor imaging in the localization of endocrine tumors [see comments]. New England Journal of Medicine $1990 \quad 323$ $1246-1249$.

13 Hofland LJ, van Koetsveld PM, Waaijers M, Zuyderwijk J, Breeman WA \& Lamberts SW. Internalization of the radioiodinated somatostatin analog [ $\left.{ }^{125} \mathrm{I}_{-} \mathrm{Tyr}^{3}\right]$ octreotide by mouse and human pituitary tumor cells: increase by unlabeled octreotide [see comments]. Endocrinology $19951363698-3706$.
14 Kwekkeboom D, Krenning EP \& de Jong M. Peptide receptor imaging and therapy. Journal of Nuclear Medicine 200041 1704-1713.

15 Schally AV \& Nagy A. Cancer chemotherapy based on targeting of cytotoxic peptide conjugates to their receptors on tumors. European Journal of Endocrinology 1999141 1-14.

16 Lamberts SW, Verleun T, Zuiderwijk JM \& Oosterom R. The effect of the somatostatin analog SMS 201-995 on normal growth hormone secretion in the rat. A comparison with the effect of bromocriptine on normal prolactin secretion. Acta Endocrinologica $1987115196-202$.

17 Londong W, Angerer M, Kutz K, Landgraf R \& Londong V. Diminishing efficacy of octreotide (SMS 201-995) on gastric functions of healthy subjects during one-week administration. Gastroenterology $198996713-722$.

18 Creutzfeldt W, Lembcke B, Folsch UR, Schleser S \& Koop I. Effect of somatostatin analogue (SMS 201-995, Sandostatin) on pancreatic secretion in humans. American Journal of Medicine 19878249 - 54.

19 Shimon I, Taylor JE, Dong JZ, Bitonte RA, Kim S, Morgan B et al. Somatostatin receptor subtype specificity in human fetal pituitary cultures. Differential role of SSTR 2 and SSTR 5 for growth hormone, thyroid-stimulating hormone, and prolactin regulation. Journal of Clinical Investigation 199799 789-798.

20 Shimon I, Yan X, Taylor JE, Weiss MH, Culler MD \& Melmed S. Somatostatin receptor (SSTR) subtype-selective analogues differentially suppress in vitro growth hormone and prolactin in human pituitary adenomas. Novel potential therapy for functional pituitary tumors. Journal of Clinical Investigation 1997 $1002386-2392$.

21 Rocheville M, Lange DC, Kumar U, Patel SC, Patel RC \& Patel YC. Receptors for dopamine and somatostatin: formation of heterooligomers with enhanced functional activity [see comments]. Science $2000 \mathbf{2 8 8} 154-157$.

22 Rocheville M, Lange DC, Kumar U, Sasi R, Patel RC \& Patel YC. Subtypes of the somatostatin receptor assemble as functional homo- and heterodimers. Journal of Biological Chemistry 2000 $2757862-7869$.

23 Ren S, Taylor JE, Culler MD \& Melmed S. Functional association of sstr 2 and sstr 5 in suppression of growth hormone secretion. Proceedings of the Endocrine Society, Denver, 2000339 (Abstract).

24 Saveanu A, Gunz G, Dufour H, Caron P, Fina F, Ouafik L et al. Bim23244, a somatostatin receptor subtype 2- and 5-selective analog with enhanced efficacy in suppressing growth hormone $(\mathrm{GH})$ from octreotide-resistant human GH-secreting adenomas. Journal of Clinical Endocrinology and Metabolism 200186 140-145.

25 Bruns C, Briner U, Lewis G, Meno-Tetang G \& Weckbecker G. SOM230: a new somatostatin peptidomimetic with broad SRIF receptor binding and a unique inhibitory profile. European Journal of Endocrinology 2002146 707-716.

26 Stroh T, Jackson AC, Sarret P, Dal Farra C, Vincent JP, Kreienkamp $\mathrm{HJ}$ et al. Intracellular dynamics of sst5 receptors in transfected COS-7 cells: maintenance of cell surface receptors during ligand-induced endocytosis. Endocrinology $2000141354-365$.

27 Aarsen RS, Bruining GJ, Grose WF, van Strik R, Lamberts SW \& Harris AG. Long-acting somatostatin analogue (Sandostatin) reduces late night insulinopenic ketogenesis in diabetic teenagers. Acta Endocrinologica Supplementum 1987286 45-53.

28 Jacobs ML, Derkx FH, Stijnen T, Lamberts SW \& Weber RF. Effect of long-acting somatostatin analog (Somatulin) on renal hyperfiltration in patients with IDDM. Diabetes Care 199720 632-636.

29 Smith LE, Kopchick JJ, Chen W, Knapp J, Kinose F, Daley D et al. Essential role of growth hormone in ischemia-induced retinal neovascularization. Science 1997276 1706-1709.

30 Grant MB, Mames RN, Fitzgerald C, Hazariwala KM, CooperDeHoff $\mathrm{R}$, Caballero $\mathrm{S}$ et al. The efficacy of octreotide in the therapy of severe nonproliferative and early proliferative diabetic retinopathy: a randomized controlled study. Diabetes Care 2000 23 504-509.

Received 4 March 2002

Accepted 4 March 2002 